\title{
Increased vesicular and vacuolar transendothelial transport in traumatic human brain oedema. A review
}

\author{
Orlando J. Castejón \\ Biological Research Institute "Drs. Orlando J. Castejón and Haydée Viloria de Castejón”, Faculty of Medicine, Zulia University, \\ Maracaibo, Venezuela
}

\begin{abstract}
The endothelial vacuolar and vesicular transports in traumatic human brain oedema have been reviewed and compared with experimental brain oedema in order to establish their role in both oedema formation and oedema resolution. Normal or "non-activated" and "activated" capillaries are found. The activated capillaries showed predominantly an enhanced abluminally orientated vesicular transport by means of small, medium and large uncoated and clathrin coated vesicles, as well as the presence of endothelial tubular structures. Activation of the endothelial nuclear zone is featured by the increased amount of micropinocytotic vesicles. Vesicles internalizing to the hypertrophic Golgi complex, lysosomes and multivesicular bodies are observed. The protein vacuolar transport is predominant in most cortical capillaries. A wide spectrum of endothelial cell mechanisms is observed increasing the vesicular and vacuolar transport, such as deep invaginations of the luminal surface, large coated vesicles, tubular structures, and transient and incomplete transendothelial channels formed either by chained plasmalemmal vesicles or elongated protein-containing vacuoles. Uncoated vesicles are seen surrounding lysosomes. Vesicular transport might be discriminated between abluminally orientated or transendothelial transport (oedema formation) and intraendothelial transport (oedema resolution) directed towards cell lysosomes to be degraded by lysosomal enzymes. The transendothelial passage via large vacuoles is mainly caused by macromolecular protein transport.
\end{abstract}

Key words: brain trauma, brain oedema, vesicular transport, vacuolar transport, endothelial mechanisms.

\section{Introduction}

Increased endothelial cell vesiculation has been earlier reported in anoxic-ischaemic lesions [30,31,36], experimental cerebral infarction [3], ionizing radiation [20], brain-stem lesion of thiamine-deficient rats [44], following intraventricular perfusion with serotonin, norepinephrine and cyclic AMP [63], ultraviolet irradiation
[49], experimental seizures [46], hypertension [28,47,59], brain trauma $[1,6,12-16,18,21,31,47,48]$, congenital hydrocephalus [10,17], and brain tumours [23,50,53-55].

The hypothesis of "vesicular transport" stands as a generally accepted process, explaining in particular macromolecular transport in capillaries [2]. The most convincing evidence to date for a vesicular transport of protein across 
reactive cerebral capillaries was earlier provided by Beggs and Waggener [1], who found that upon reproducible contusion of the spinal cord in cats, horseradish peroxidase escaped from the vessels by means of pleomorphic vesicles, tubules and large vacuoles.

The orientation of pinocytotic vesicles correlating with the stage of the oedematous process was first made by Wagner et al. [61], and reproduced by Cervós-Navarro et al. [19]. Sazaki et al. [49] reported a typical orientation of the micropinocytotic vesicles depending upon the stage of the oedematous process: in the early stages, they are found preferably at the luminal side of endothelial cells, and later on they predominate at the abluminal endothelial cell membrane. Ferszt et al. [29] reported diffuse macro- and micropinocytotic activity in focal brain oedema due to ultraviolet irradiation as tissue water content reaches maximum values.

Casley-Smith and Carter [4] found that large vacuoles in inflamed endothelium do indeed transport macromolecules across the cells. In addition, large endothelial vacuoles and transendothelial vacuolar transport have been described in a large variety of pathological entities, such as brain tumours [50,53-55], anoxic-ischaemic lesions [6,8-10,13,17,21,27,30,33,34, $40,41,45$ ], injections of Gram-negative endotoxin [24], lead encephalopathy [39], experimental allergic encephalomyelitis [35], and human brain oedema [6,8]. These studies show that the formation of endothelial vacuoles could be interpreted as opening of the endothelial 'pore system' induced by different pathological conditions [6]. Lossinsky et al. [42,43] studied the vesicular and canalicular transport structures in endothelial cells after crude leptomeningeal damage in mice, and in cold lesion injury in cats. Castejón [6-9] described an increased vesicular and vacuolar transendothelial transport, and formation of transendothelial channels in endothelial cells and pericytes in human traumatic and complicated brain injuries. Shibata et al. [50] reported increased endothelial pinocytotic vesicle formation in human brain tumours. Claudio et al. [25] found an increased endothelial cell vesicular transport in rat experimental autoimmune encephalomyelitis. According to these authors, immunogold staining of endogenous albumin demonstrated the presence of albumin in cytoplasmic vesicles and in channel-like tubular structures adjacent to endothelial cell junctions. These results indicated that there is a role for vesicles in transendothelial cell transport and oedema formation in animals with experimental autoimmune encephalomyelitis. Takano et al. [53] ob- served an increased vesicular transport in human glioma capillaries. Kato et al. [38] demonstrated increased numbers of vesicles and vacuoles in endothelial brain capillaries in cerebral oedema from fulminant hepatic failure.

Zumkeller and Dietz [65] described an increased protein vesicular transport in rats after treatment with nimodipine. Cicciarello et al. [26] found enhanced vesicular transport of horseradish peroxidase in an experimental model of whole brain after irradiation. Plateel et al. [45] examined the increased permeability of albumin after hypoxia, attributed to non-specific vesicular transport in a cell culture model of brain barrier.

Hofman et al. [37] showed an increased vascular permeability for plasma proteins in vivo induced by vascular endothelial growth factor-A (VEGF) in blood-retinal barrier endothelium predominantly caused by a mechanism involving active trans-endothelial transport via pinocytotic vesicles, and not by formation of endothelial fenestrations or vesiculo-vacuolar organelles.

Castejón et al. [15] reported an increased vesicular and vacuolar transendothelial transport in two patients with post-traumatic seizures. Lossinsky et al. [42] examined the vesicular and canalicular transport structures in the injured mammalian blood-brain barrier. Lossinsky and Shivers [43] reviewed in detail the structural pathways for macromolecular and endothelial cell transport during inflammatory conditions and brain injuries. Cipolla et al. [27] found increased apical and basolateral pinocytosis in cerebral endothelium during ischaemia/reperfusion, and elevated intravascular pressure. Castejón $[10,17]$ described an increased vesicular and vacuolar transport in cortical capillaries from parietal and frontal cortex of patients with congenital hydrocephalus and Arnold-Chiari malformation.

Vesicular and vacuolar transport is a highly dynamic process currently studied in experimental animal works with electron dense tracers. The study of endothelial vesicular and vacuolar transport in human cortical biopsies is obviously limited for many reasons, such as: a) ethical principles that limit the use of electron dense probes in vivo, b) samples randomly obtained, c) ultrathin sections giving isolated images of endothelial cell substructures, d) great variability of the nature of traumatic agents and impact energy, and the patient's age and state of health.

In the present review we describe the morphological evidence of increased vesicular and vacuolar transports induced by human traumatic brain injuries. Emphasis will be placed on the role played by vesicular and vac- 
uolar transports in oedema formation and oedema res olution. The variety of endothelial cell mechanisms observed in severe vasogenic brain oedema also are analysed.

\section{Vesicular and vacuolar transendothelial transport as morphological signs of increased cerebrovascular permeability}

\section{Resting cortical capillaries}

In traumatic moderate brain oedema, some gray matter cortical capillaries exhibit a normal appearance with an inactive, resting endothelial cytoplasmic peripheral zone, characterized by straight, almost smooth, luminal plasma membrane, without pseudopodic expansions and scarce micro- and macropinocytotic activity. In these normal capillaries, the basement membrane appears as a compact structure formed by filaments embedded in a homogeneous matrix. Only some free and membrane-bound clathrin-coated vesicles are encountered. The endothelial junctions are structurally intact, and the astrocytary glial end-feet appear intimately applied to the basement membrane $[6,8,11]$. These "non-leaky" capillaries are not activated by the mechanical injury exerted by the intensity of brain trauma.

\section{Activated cortical capillaries}

Other capillaries are activated by the traumatic injury and show an endothelial cell luminal surface activity, exhibiting abundant luminal microvilli, presence of coated vesicles, remarkable orientation of numerous abluminal uncoated pinocytotic vesicles toward the capillary basement membrane (Fig. 1), and a lesser amount of caveolae intracellularis or 'pits' connected to the luminal endothelial surface, suggesting abluminally orientated vesicular transport $[6,8]$. The non-leaky segments of capillaries contain "immobile" endothelial vesicles [58], which are activated in the leaky segments by the impact energy.

Chained pinocytotic vesicles fused with each other and with tubular invaginations to form shuttle vesicles or incomplete transient transendothelial channels $[7,42,43]$. In addition, large spheroid or elongated vacuoles, containing hematogenous oedema fluid appear free in the endothelial cytoplasm (Figs. 2 and 3). These vacuoles discharge their content directly into the basement membrane or by means of plasmalemmal vesicles. The basement membrane appears swollen, and abundant proteinaceous oedema fluid is seen separating the basement membrane from the perivascular glycogen-rich and glycogen depleted astroglial end-feet $[11,14]$. The latter are remarkably swollen and their limiting membranes appear fragmented.

Some actin-like filaments are attached to the vacuole limiting membrane, suggesting that the cytoskeleton is involved in the transendothelial movement of these vacuoles [8]. In normal capillaries the small vesicular and vacuolar transport is a random process powered by Brownian motion [4], but in the case of an enhanced cerebrovascular permeability, the participation of cytoskeletal structures is required to speed up the vacuolar and vesicular transport. A detailed study of the longitudinal sections of the capillary wall show that, in addition to the endothelial peripheral zone, the nuclear and organelle endothelial zones (Fig. 4) also

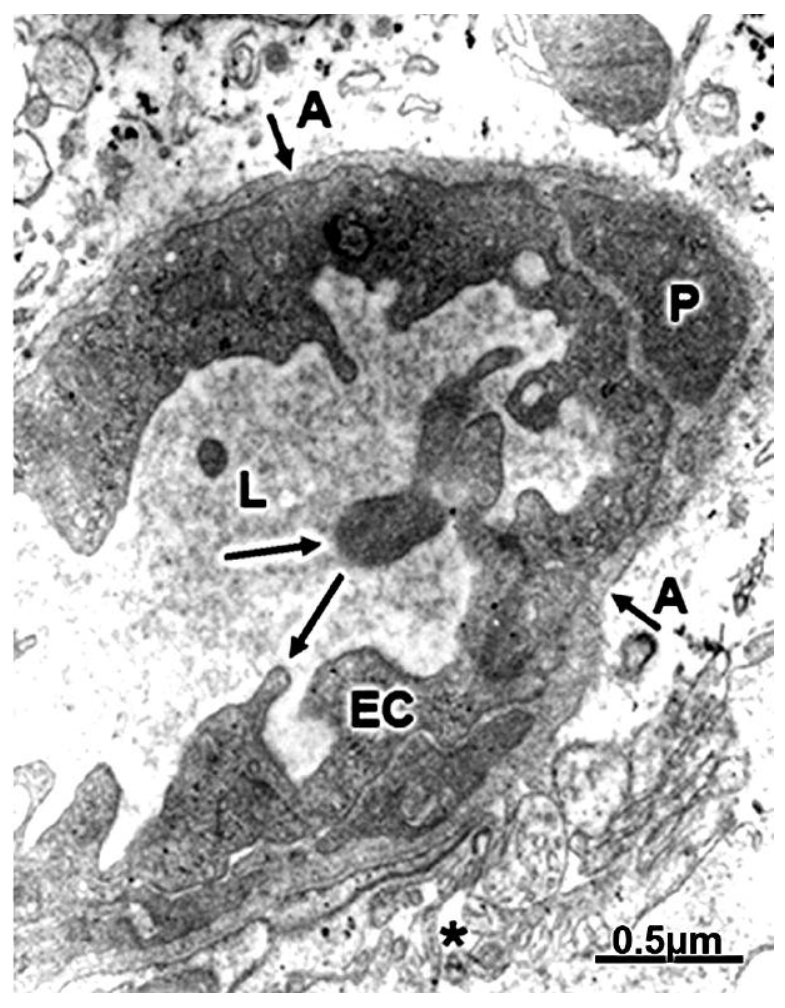

Fig. 1. Brain trauma. Subdural haematoma. Left parietal cortex. Cross section of a traumatically activated cortical capillary showing prominent protrusion of pseudopodic endothelial cell (EC) expansions (long arrows) engulfing the proteinaceous oedema fluid from the capillary lumen (L). The apparently normal basement membrane (short arrows), the enclosed pericital cytoplasm $(P)$, and the astrocytic perivascular end-feet $(A)$ also are seen. The asterisk labels the enlarged perivascular space. 


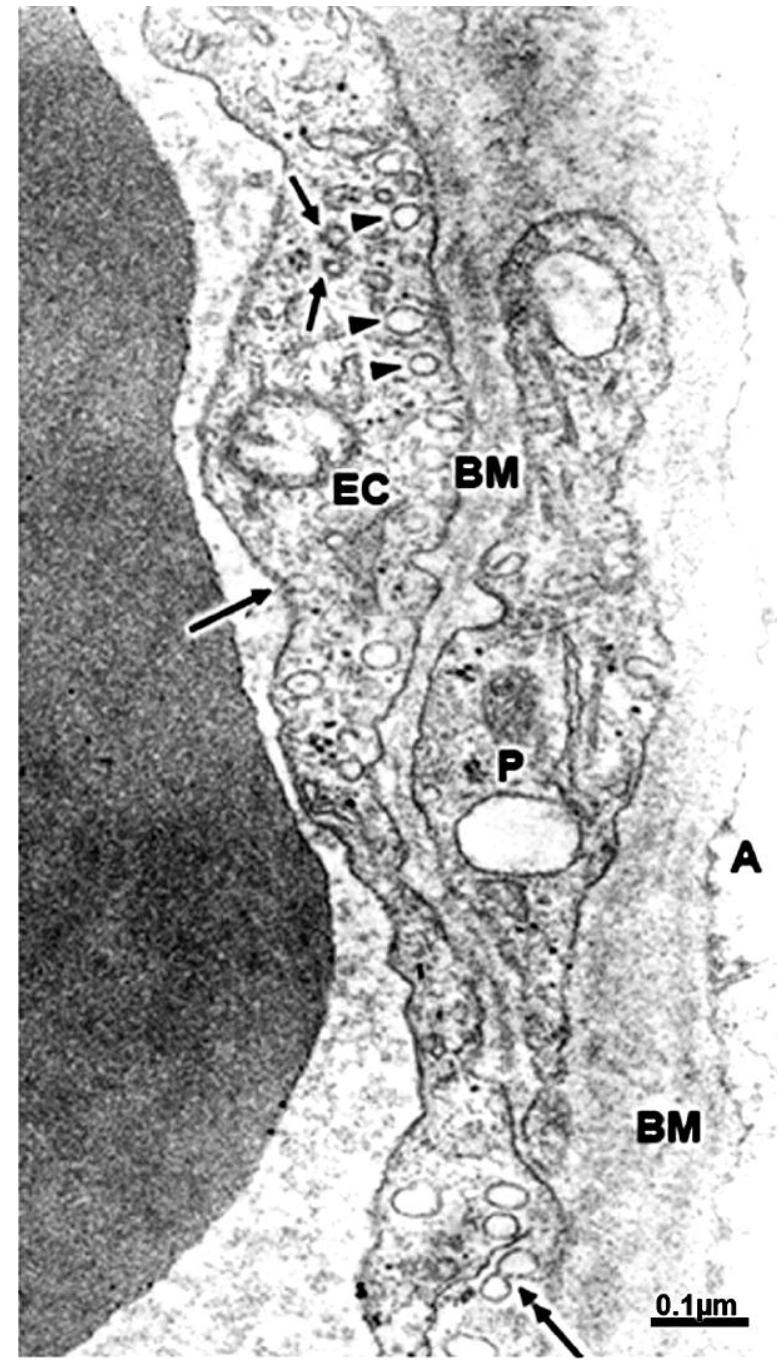

Fig. 2. Brain trauma. Right epidural haematoma. Right temporal cortex. Endothelial peripheral cytoplasm (EC) showing predominant vesicular transport (arrowheads) polarized toward the swollen basement membrane (BM). A caveolae intracellularis (long arrow) and fused micropinocytotic vesicles (double head arrow) are seen. The swollen pericyte $(P)$ and basement membrane $(B M)$ are noted. The limiting membrane of astrocytic (A) perivascular end-foot appears irregularly applied to the basement membrane outer surface.

exhibit surface activity, with shallow and deep invaginations of luminal plasma membrane to form proteintransporting vacuoles [6,8]. Deep invaginations of endothelia cell luminal membranes were also reported by Lossinski et al. [42] in experimental models of brain

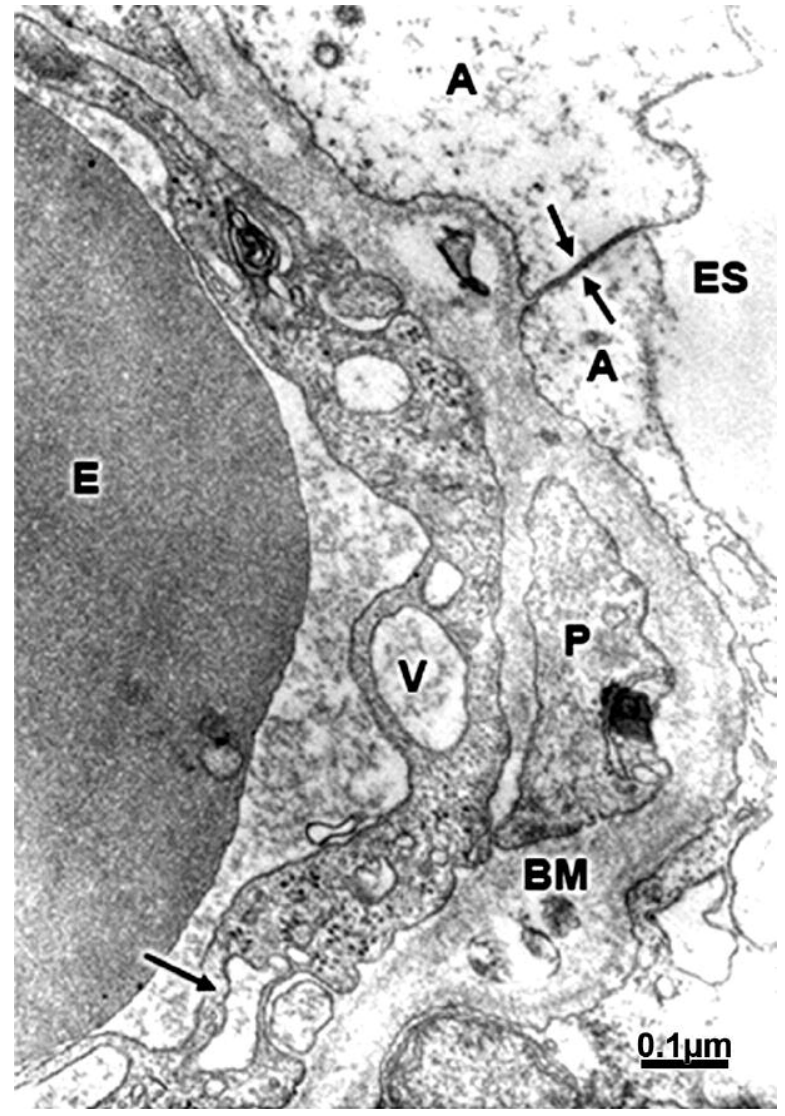

Fig. 3. Brain trauma. Subdural haematoma. Left parietal cortex. Endothelial cell peripheral zone showing large round $(\mathrm{V})$ and elongated (long arrow) vacuoles. An erithrocyte (E), the swollen and vacuolated basement membrane, the oedematous astrocytic perivascular end-feet (A), and the distended extracellular space (ES) also are distinguished. The short arrows indicate the astrocytic end-feet gap junction.

injury. The endothelial cell nuclear zone in normal capillaries shows a little or no micropinocytotic activity $[6,51,52]$.

Vacuoles and vesicles actually participating in the transendothelial transport toward the basement membrane could be distinguished from those internalizing to some cell organelles, such as multivesicular bodies, Golgi apparatus and lysosomes (Figs. 5 and 6). The Golgi complex appears hypertrophic with dilated endoplasmic sacs and Golgi vacuoles. Deep invaginations of the luminal surface are formed in the vicinity of the Golgi formation phase, and numerous clathrin-coated and uncoated vesicles are observed in this area [8]. 


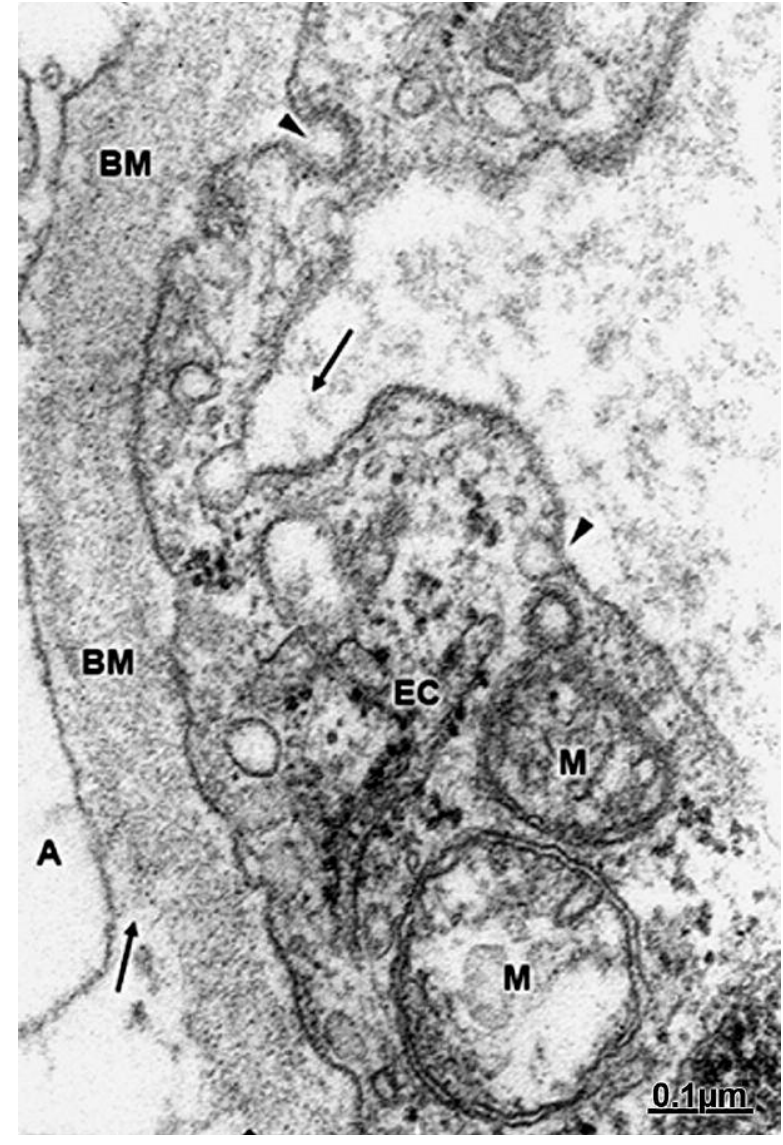

Fig. 4. Brain trauma. Rigt parieto-temporal subdural haematoma. Endothelial cell (EC) organelle and peripheral zones showing the formation of pinocytotic and clathrin-coated vesicles (arrowheads). A deep invagination of the endothelial luminal membrane (long arrow) ends in a micropinocytotic vesicle. Note the swollen mitochondria $(M)$, the rough endoplasmic reticulum (ER), and the perivascular astrocytic end-foot (A) dissociated (arrow) from the thickened and rarefacted basement membrane (BM).

Golgi vesicles are the structural vectors of the recycled membranes [51,52]. Presumably a considerable amount of Golgi complex-derived membrane is inserted into the endothelial luminal plasmalemma to provide new membranes for the increased vesicular and vacuolar transport. This sustained over-function might induce Golgi complex hypertrophy $[8,16]$.

Some pinocytotic vesicles are found surrounding dense cored vesicles and small lysosomes, suggesting that some amount of plasma protein is transported by plasmalemmal vesicles towards the lysosomes to be

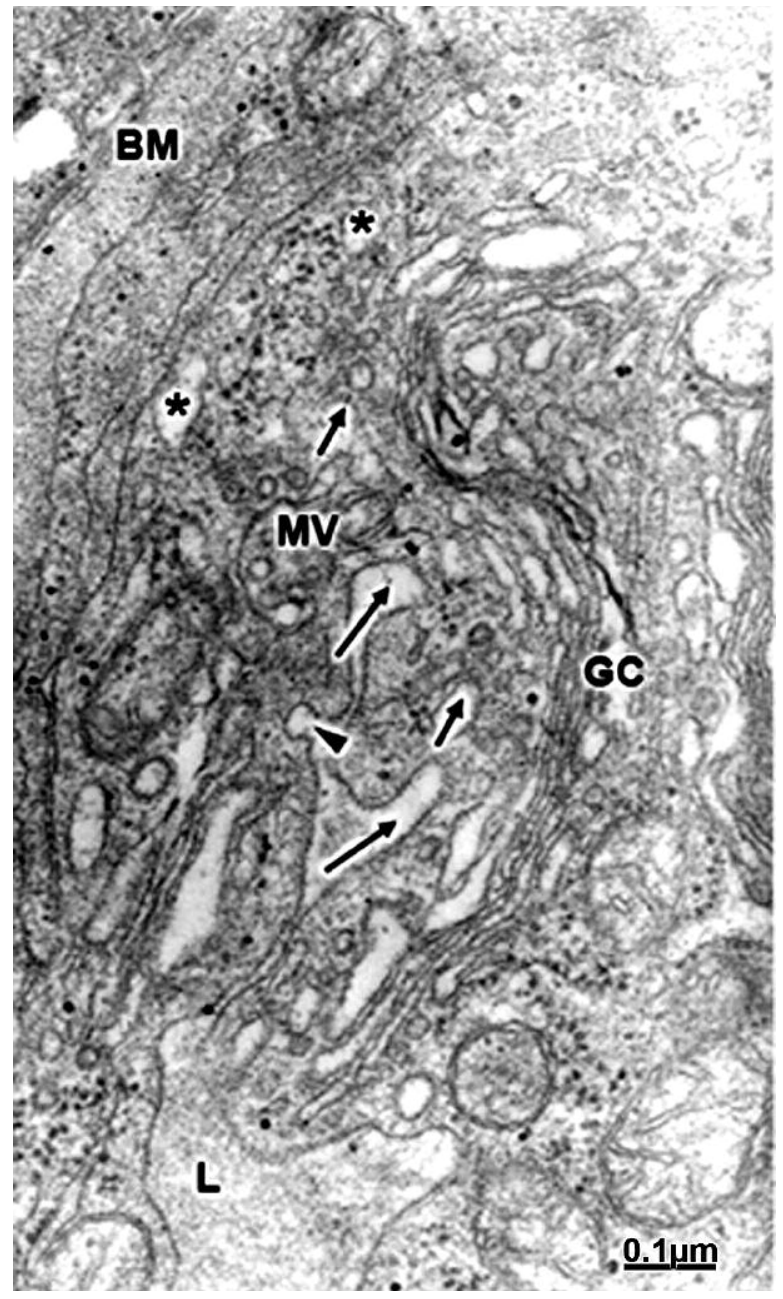

Fig. 5. Brain trauma. Contusion of frontal region. Right frontal cortex. Deep invaginations of the endothelial cell luminal membrane (long arrows) are formed at the endothelial peripheral cytoplasm. A caveolae intracellularis (arrowhead) is observed at the endothelial cell luminal membrane. Some coated and uncoated vesicles and vacuoles are internalized (small arrows) towards the hypertrophic Golgi complex (GC). Note that other endothelial vacuoles (asterisks) follow a transcapillary route toward the basement membrane (BM). Some vesicles appear surrounding a multivesicular body (MB). Note also the hydropic changes of Golgi endoplasmic sacs and vacuoles. The capillary lumen $(\mathrm{L})$ also is noted.

degraded by lysosomal enzymes, as an endothelial cell mechanism of oedema resolution [8,58]. At the Golgi region, uncoated and coated pinocytotic vesicles and microtubules appear topographically related to lyso- 


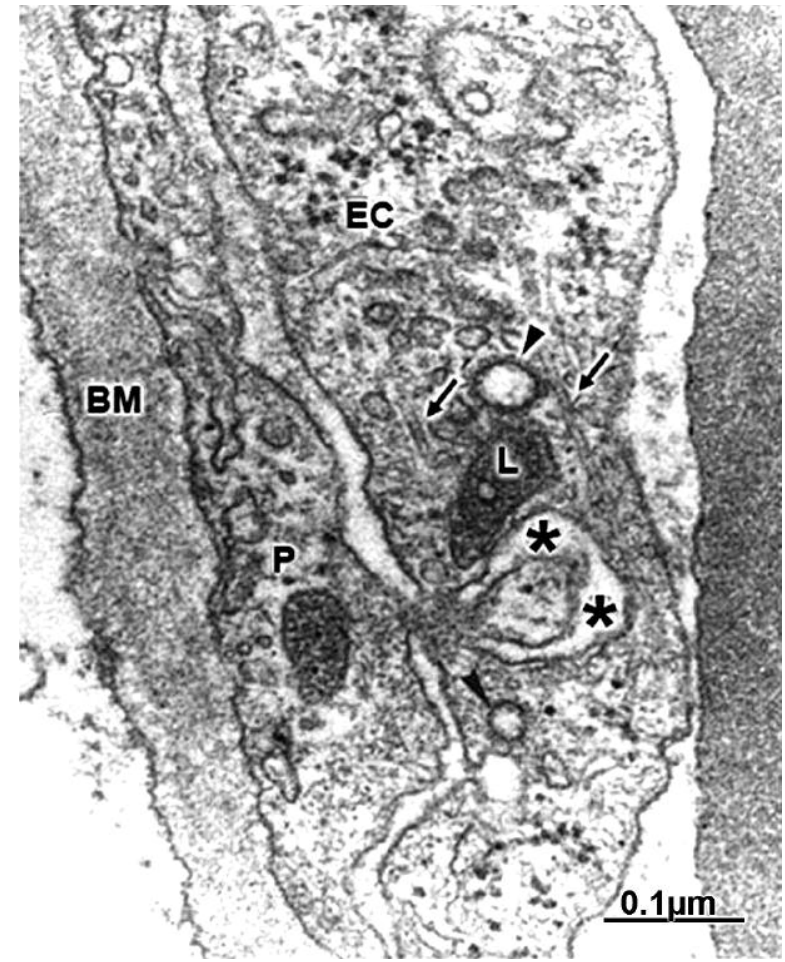

Fig. 6. Brain trauma. Epidural haematoma. Right temporal cortex. Endothelial peripheral cytoplasm (EC) showing a clathrin-coated vesicle (arrowhead) and microtubules (short arrows) in topographic relationship with a lysosome (L). A dilated curved basement membrane bifurcation (asterisks) appears in a sublemmal location. The pericyte cell $(P)$ and the swollen basement membrane (BM) also are observed.

somes (Fig. 6). Coated pits and clathrin-coated vesicles are also seen.

These electron microscopic findings suggest that endothelial vesicular transport can be discriminated between abluminally orientated transendothelial transport toward the capillary basement membrane inducing oedema formation, and cell organelle orientated intraendothelial vesicular transport directed towards Golgi complex, lysosomes and multivesicular bodies for oedema resolution [8]. Van Deurs [58] has also shown evidence of transport of exogenous material to lysosomes by uncoated endothelial vesicles to be degraded by hydrolytic enzymes.

Numerous free and abluminal membrane-bound pinocytotic vesicles and vacuoles are also found discharging their content into the capillary basement membrane, suggesting the orientated increased pino-

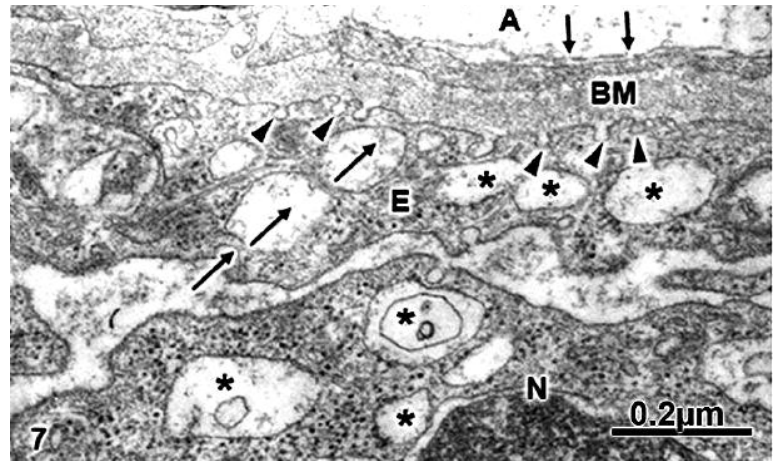

Fig. 7. Brain trauma. Fronto-parieto-occipital subdural haematoma. Left paritetal cortex. Activated endothelial cytoplasm of nuclear $(\mathrm{N})$ and peripheral zones showing increased vacuolar and vesicular transports. The large arrows indicate the direction of vacuolar transcapillary passage. The dilated rough endoplasmic reticulum also is vacuolated (asterisks). There is a clear contraluminal orientation of micropinocytotic transport (arrowheads) since the micropinocitotic vesicles appear discharging their content to the swollen basement membrane (BM). Note the disrupted limiting membrane (short arrows) of the swollen perivascular astrocytic end-foot (A).

cytotic and vacuolar transport toward the tissue front (Fig. 7). These capillaries display notably oedematous pericytes also with an increased vesicular and vacuolar transport $[9,18]$, and swollen glycogen-rich and glycogen-depleted astrocytic perivascular end-feet [14], indicating the route followed by the hematogenous oedema fluid.

Some endothelial vacuoles are formed by a wellknown mechanism of emission of microvilli, which initially projects towards the capillary lumen and afterwards refolds over the neighbouring endothelial luminal surface, or either by interdigitation of a pseudopodic expansion and a microvillus [5,6] (Fig. 8).

In relationship to the identification of endothelial vacuoles, much confusion has been caused in electron microscopy by ultrathin sections of simple indentations of endothelial cell plasma membrane of peripheral cytoplasmic zone, by phagocytosis, and by dilated endoplasmic reticulum [5]. In our studies we have clearly differentiated between these possibilities, and considered that unidirectional transendothelial passage of true protein-containing vacuoles is primarily concerned with oedema formation $[6,8]$. 


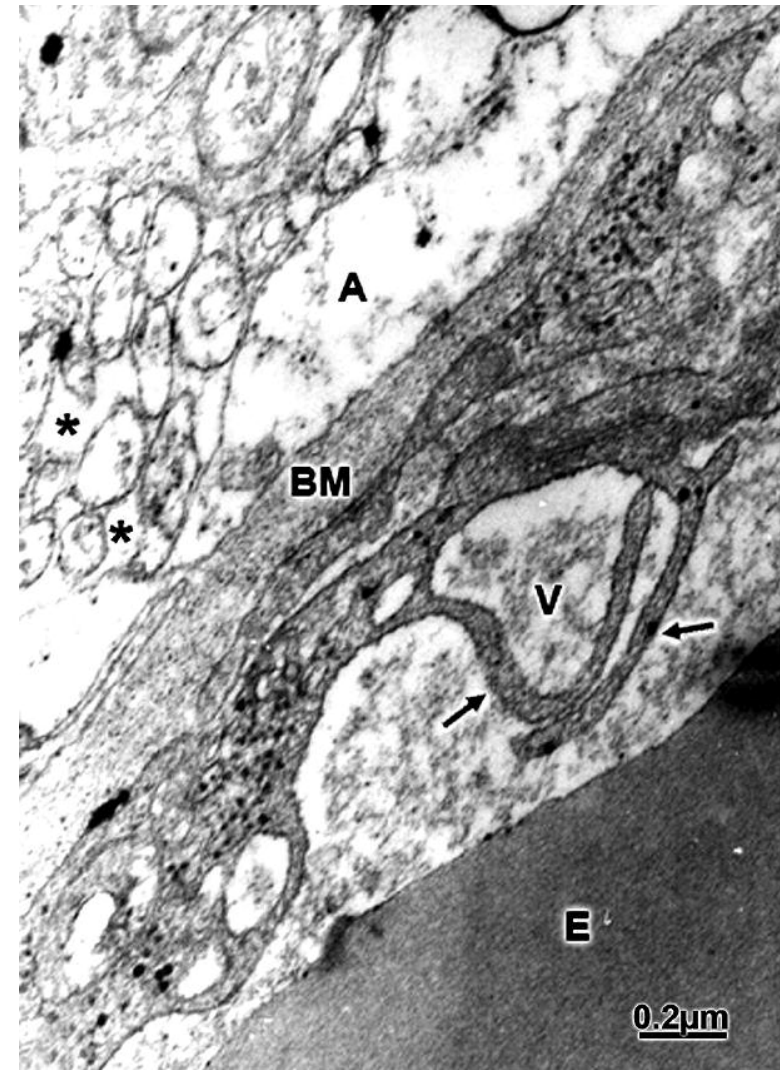

Fig. 8. Brain trauma. Parieto-temporal subdural haematoma. Left parietal cortex. Endothelial cell peripheral zone showing emission of two microvilli (arrows) entrapping haematogenous oedema fluid, and forming protein-containing vacuoles, as the most frequent mechanism of oedema formation. An erythrocyte (E), the basement membrane (BM), the swollen astrocytic end-foot (A), and the enlarged extracellular space (asterisks) also are seen.

In some capillaries, the cytoplasm of the endothelial peripheral zone becomes so attenuated that a large micropinocytotic vesicle and vacuoles can function almost as incomplete and transient transendothelial channels [6] (Fig. 9).

Earlier studies on experimental brain trauma have demonstrated that protein leakage occurs as early as three (3) minutes after brain injury [48]. In addition, plasma protein extravasation has been reported ten minutes after impact injury in spinal gray matter microvasculature [31]. In traumatic human brain injuries we have shown evidence that there is an accumulation of proteinaceous oedema fluid in the interspace

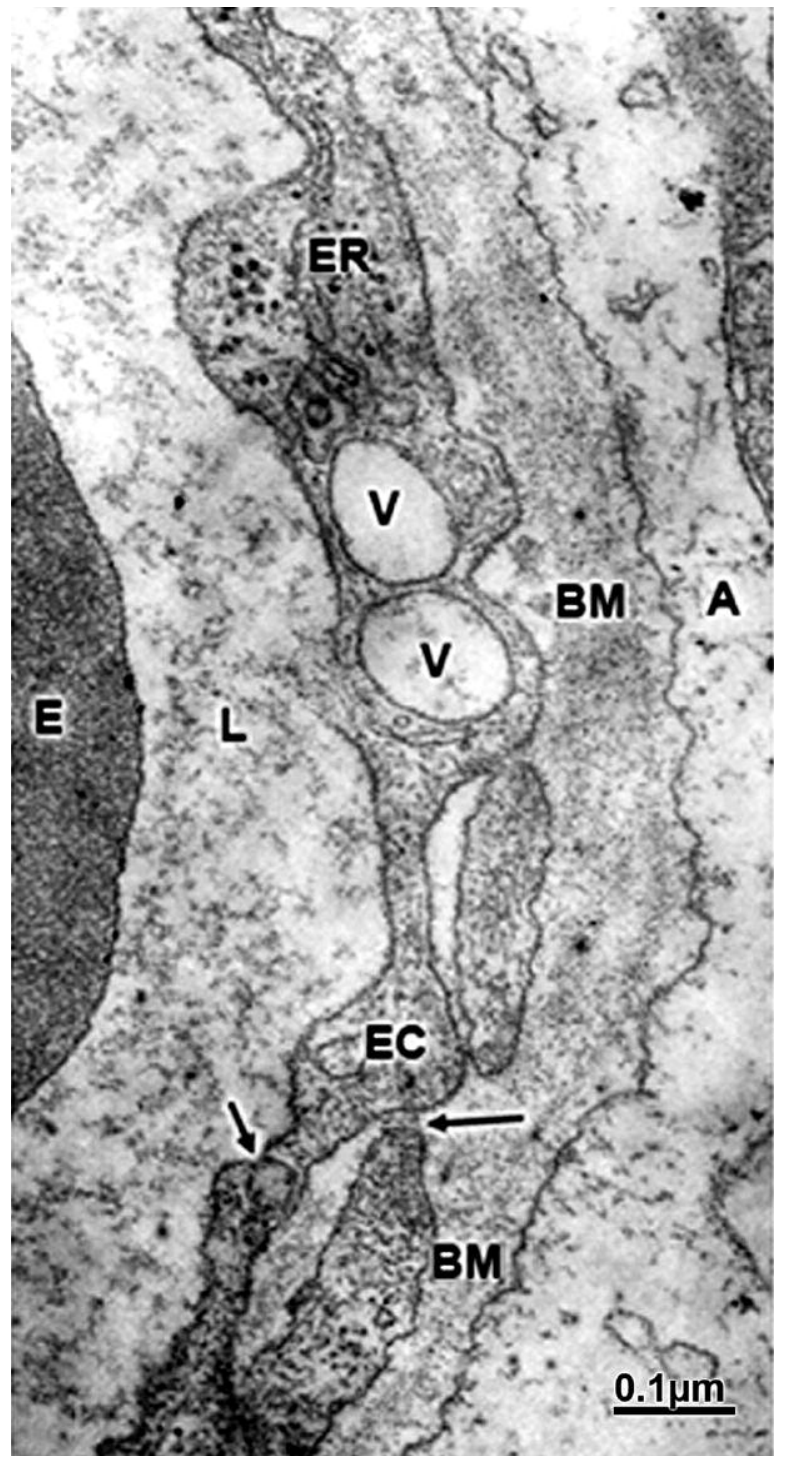

Fig. 9. Brain trauma. Subdural haematoma. The attenuated endothelial peripheral zone (EC) with a large micropinocytotic vesicle (small arrow) and vacuoles (V). An erythrocyte (E), the basement membrane (BM), the endoplasmic reticulum (ER), the swollen astrocytic endfoot $(A)$, the vessel lumina $(L)$.

between the basement membrane and the astrocytary perivascular end-feet twenty four (24) hours after a severe brain injury [8,11-13]. Extravasation of proteinaceous oedema fluid in moderately oedematous regions is mainly due to the enhanced vesicular and vacuolar transport because most tight endothelial junctions are intact and structurally closed [8,22]. 


\section{Concluding remarks}

The endothelial vacuolar and vesicular transports have been studied in traumatic brain injuries in order to establish their role in both oedema formation and oedema resolution. Normal or "non-activated" and "activated" capillaries are found. The activated capillaries show predominantly an enhanced abluminally orientated vesicular transport by means of small, medium and large uncoated and clathrin-coated vesicles, as well as the presence of endothelial tubular structures. Vesicles internalizing to the hypertrophic Golgi complex, lysosomes and multivesicular bodies are observed for oedema resolution. The vacuolar transport is predominant in most cortical capillaries. The basic endothelial cell mechanisms found in complicated human brain injuries are deep invaginations of luminal surface, formation of large coated vesicles, tubular structures, and transient and incomplete transendothelial channels formed either by chained plasmalemmal vesicles or elongated protein-containing vacuoles. Uncoated vesicles are seen surrounding lysosomes. The endothelial vesicular transport can be discriminated between abluminally orientated or transendothelial transport (oedema formation), and intraendothelial transport (oedema resolution) directed towards multivesicular bodies and lysosomes. The transendothelial passage via large vacuoles is mainly due to protein transport. Most endothelial junctions examined in moderate oedematous regions are structurally intact.

\section{References}

1. Beggs JJ, Waggener JD. Transendothelial vesicular transport of protein following compression injury to the spinal cord. Lab Invest 1976; 34: 428-439.

2. Bundgaard M, Frokjaer-Jensen J, Crone C. Endothelial plasmalemmal vesicles as elements in a system of branching invaginations from the cell surface. Proc Natl Acad Sci USA 1979; 76: 6439-6442.

3. Calhoun CC, Mottaz JA. Capillary bed of rat cerebral cortex. The fine structure in experimental cerebral infarction. Arch Neurol 1966; 15: 320-328.

4. Casley-Smith JR, Carter DB. The passage of macromolecules across inflamed capillary endothelium via large vacuoles. Microvasc Res 1979; 18: 319-324.

5. Casley-Smith JR. The functioning and interrelationships of blood capillaries and lymphatics. Experientia 1976; 32: 1-12.

6. Castejón OJ. Electron microscopic study of capillary wall in human cerebral edema. J Neuropathol Exper Neurol 1980; 49: 296 328.

7. Castejón OJ. Formation of transendothelial channels in traumatic human edema. Pathol Res Pract 1984; 179: 7-12.
8. Castejón OJ. Increased vesicular and vacuolar transport in traumatic human brain edema. A combined electron microscopy and theoretical approach. J Submicrosc Cytol 1984; 16: 359-369.

9. Castejón OJ. Submicroscopic changes of cortical capillary pericytes in human perifocal brain edema. J Submicrosc Cytol 1984; 16: 601-618.

10. Castejón OJ. Transmission electron microscope study of human hydrocephalic cerebral cortex. J Submicrosc Cytol Pathol 1994; 26: 29-39.

11. Castejón OJ. Ultrastructural alteration of human cortical capillary basement membrane in perifocal brain edema. J Submicrosc Cytol Pathol 1988; 20: 519-536.

12. Castejón OJ. Electron microscopic analysis of cortical biopsies in patients with traumatic brain injuries and dysfunction of neurobehavioural system. J Submicrosc Cytol Pathol 1998; 30: 145-156.

13 Castejón OJ, Castejón HV, Diaz M, Castellano A. Consecutive light microscopy, scanning-transmission electron microscopy and transmission electron microscopy of traumatic human brain oedema and ischaemic brain damage. Histol Histopathol 2001; 16: 1117-1134.

14. Castejón OJ, Diaz M, Castejón HV. Castellano A. Glycogen-rich and glycogen-depleted astrocytes in the oedematous human cerebral cortex associated with brain trauma, tumours and congenital malformation: an electron microscopy study. Brain Inj 1988; 16: 109-132.

15. Castejón OJ, Castejón HV, Zavala M, Sanchez ME, Diaz M. A light and electron microscopic study of oedematous human cerebral cortex in two patients with post-traumatic seizures. Brain Inj 2002; 16: 331-346.

16. Castejón OJ. Electron Microscopy of Human Brain Edema. Astrodata, Maracaibo 2008.

17. Castejón OJ. Blood-brain barrier ultrastructural alterations in human congenital hydrocephalus and Arnold-Chiari malformation. Folia Neuropathol 2009; 47: 11-19.

18. Castejón OJ. Ultrastructural pathology of cortical capillary pericytes in human traumatic brain oedema. Folia Neuropathol 2011; 49: 162-173.

19. Cervós-Navarro J, Christmann V, Sasaki S. An ultrastructural substrate for the resolution of postirradiation brain edema. In: Pappius HM, Fein-del W (eds.). Dynamics of Brain Edema. SpringerVerlag, Berlin 1976; pp. 43-49.

20. Cervós-Navarro J. Brain edema due to ionizing radiation. In: Klatzo I, Seitelberger F (eds.). Brain Edema. Springer-Verlag, New York 1967; pp. 632-638.

21. Cervós-Navarro J, Lafuente JV. Traumatic brain injuries: structural changes. J Neurol Sci (Suppl.) 1991; S3-S14.

22. Cervós-Navarro J, Turker T, Worthmann F. Morphology of non-vascular intracerebral fluid spaces. Acta Neurochir (Wien) (Suppl.) 1994; 60: 147-150.

23. Challa VR, Moody DM, Marshall RB, Kelly DL Jr. The vascular component in meningiomas associated with severe cerebral edema. Neurosurgery 1980; 7: 363-368.

24. Clawson CC, Hartman J, Vernier RL. Electron microscopy of the effect of gram-negative endotoxin on the blood-brain barrier. J Comp Neurol 1966; 127: 183-198.

25. Claudio L, Kress Y, Norton WT, Brosnan CF. Increased vesicular transport and decreased mitochondrial content in blood-brain barrier endothelial cells during experimental autoimmune encephalomyelitis. Am J Pathol 1989; 135: 1157-1168. 
26. Cicciarello R, D'Avella D, Gagliardi ME, Albiero F, Vega J, Angileri FF, D'Aquino A, Tomasello F. Time-related ultrastructural changes in an experimental model of whole brain irradiation. Neurosurgery 1996; 38: 772-780.

27. Cipolla MJ, Crete R, Vutuio L, Rix RD. Transcellular transport as a mechanism of blood-brain barrier disruption during stroke. Front Biosci 2004; 9: 777-785.

28. Dinsdale HB. Hypertension and the blood-brain barrier. In: Cervos-Navarro J, Betz E, Ebhardt G, Ferst R, Wullemweber R (eds.). Advances in Neurology. Vol. 20. Raven Press, New York 1978; pp. 341-346.

29. Ferszt R, Cervós-Navarro J, Sasaki S, Neu S, Brock M. Vesicular transport through endothelial cells in focal brain edema. In: Cervós Navarro J, Betz E, Ebhardt G, Ferst R, Wullemweber R (eds.). Advances in Neurology. Vol. 20. Raven Press, New York 1978; pp. 341-346.

30. Garcia JH, Lossinsky AS, Nishimoto K, Klatzo I, Light-Foote Jr W. Cerebral microvasculature in ischemia. In: Cervós-Navarro J, Betz E, Ebhardt G, Ferst R, Wullemweber R (eds.). Advances in Neurology. Vol. 20. Raven Press, New York 1978; pp. 141-148.

31. Griffiths IR. Ultrastructural changes in spinal gray matter microvasculature after impact injury. In: Cervos-Navarro J, Betz E, Ebhardt G, Ferst R, Wullemweber R et al. (eds.). Advances in Neurology. Vol. 20. Raven Press, New York 1978; pp. 415-422.

32. Hills CP. Ultrastructural changes in the capillary bed of the rat cerebral cortex in anoxic-ischemic brain lesions. Am J Pathol 1964; 44: 531-544.

33. Hirano A. The fine structure of brain in edema. In: Bourne G (ed.). The Structure and Function of Nervous Tissue. Vol. II. Academic Press, New York 1969; pp. 69-135.

34. Hirano A. Further observations on the fine structure of pathological reaction in cerebral blood vessels. In: Cervós-Navarro J, Betz E, Matakas F (eds.). The Cerebral Vessel Wall. Vol. 2. Raven Press, New York 1976; pp. 41-49.

35. Hirano A, Dembitzer HM, Becker NH, Levine J, Zimmerman HM. Fine structural alterations of the blood-brain barrier in experimental allergic encephalomyelitis. J Neuropathol Exp Neurol 1970; 24: $432-$ 440.

36. Hirano A, Kawanami T, Llena JF. Electron microscopy of the bloodbrain barrier in disease. Microsc Res Tech 1994; 27: 543-556.

37. Hofman P, Blaauwgeers HG, Tolentino MJ, Adamis AP, Nunes Cardozo BJ, Vrensen GF, Schlingemann RO. VEGF-A induced hyperpermeability of blood-retinal barrier endothelium in vivo is predominantly associated with pinocytotic vesicular transport and not with formation of fenestrations. Vascular endothelial growth factor-A. Curr Eye Res 2000; 21: 637-645.

38. Kato M, Hughes RD, Keays RT, Williams R. Electron microscopic study of brain capillaries in cerebral edema from fulminant hepatic failure. Hepatology 1992; 15: 1060-1066.

39. Lampert P, Garro F, Pentschew A. Lead encephalopathy in suckling rats. An electron microscopic study. In: Klatzo I, Seitelberger F (eds.). Brain Edema. Springer-Verlag, New York 1967; pp. 207-222.

40. Long DM, Hartman JF, French L. The ultrastructure of human cerebral edema. J Neuropathol Exp Neurol 1966; 25: 373-395.

41. Long DM. Microvascular changes in cold injury edema. In: Go KG, Baethmann A (eds.). Recent Progress in Study and Therapy of Brain Edema. Plenum Press, New York 1982; pp. 45-54.
42. Lossinsky AS, Vorbrodt AW, Wisniewski HM. Ultracytochemical studies of vesicular and canalicular transport structures in the injured mammalian blood-brain barrier. Acta Neuropathol 1983; 61: 239-245

43. Lossinsky AS, Shivers RR. Structural pathways for macromolecular and cellular transport across the blood-brain barrier during inflammatory conditions. Review. Histol Histopathol 2004; 19: 535-564.

44. Manz HJ, Robertson DM. Vascular permeability to horseradish peroxidase in brainstem lesions of thiamine deficient rats. Am J Pathol 1972; 66: 565-575.

45. Plateel M, Teissier E, Cecchelli R. Hypoxia dramatically increases the nonspecific transport of blood-borne proteins to the brain. J Neurochem 1997; 68: 874-877.

46. Petito CK, Schaefer JA, Plun F. Ultrastructural characteristics of the brain and blood-brain barrier in experimental seizures. Brain Res 1977; 127: 251-267.

47. Povlishock JR, Kontos HA, Wei EP, Rosemblum WD, Becker DP. Changes in the cerebral microvasculature after hypertension and trauma: A combined scanning and transmission electron microscopic analysis. The Cerebral Microvasculature. Investigations of the Blood-Brain Barrier. In: Eisenberg M, Suddith RL (eds.). Advances Experimental and Medical Biology. Vol. 131. Plenum Press, New York 1980; pp. 227-238.

48. Povlishock JT, Becker DP, Sullirang HC, Miller JD. Vascular permeability alterations to horseradish peroxidase in exprimental brain injury. Brain Res 1978; 153: 223-239.

49. Sasaki S, Fertszt R, Cervos-Navarro J. Transendothelial vesicular transport of protein in brain edema induced by ultraviolet irradiation. Acta Neuropathol 1977; 40: 207-212.

50. Shibata S, Fukushima M, Inoue M, Tsutsumi K, Mori K. Ultrastructure of capillary permeability in human brain tumors. Part 1: Gliomas associated with cerebral edema. No Shinkei Geka 1985; 13: $275-281$.

51. Simionescu M, Simionescu N, Palade GE. Morphometric data on the endothelium of blood capillaries. J Cell Biol 1974; 60: 128-152.

52. Simionescu M, Simionescu N, Palade GE. Differentiated microdomains on the luminar surface of capillary endothelium: distribution of lectin receptors. J Cell Biol 1982; 94: 406-413.

53. Takano S, Yoshii Y, Nose T. Ultrastructure of glioma vessel - morphometric study for vascular permeability. No To Shinkei 1991; 43: 49-56.

54. Torack RM. Ultrastructure of capillary reaction to brain tumors. Arch Neurol 1961; 5: 416-428.

55. Torack RM. Ultrastructure of capillary reaction to brain tumors. Arch Neurol 1961; 5: 86-98.

56. Van Deurs B. Endocytosis in high endothelial venules. Evidence for transport of exogenous material to lysosomes by uncoated 'endothelial' vesicles. Microvasc Res 1978; 16: 280-293.

57. Van Deurs B, Amtorp O. Blood-brain barrier in rats to the hemepeptide microperoxidase. Neuroscience 1978; 3: 737-748.

58. Van Deurs B. Endocytosis in high-endothelial venules. Evidence for transport of exogenous material to lysosomes by uncoated endothelial vesicles. Microvasc Res 1978; 16: 280-293.

59. Van Deurs B. Observations on the blood-brain barrier in hypertensive with particular references to phagocytic pericytes. J Ultrastruct Res 1976; 56: 65-77. 
60. Vankov V. Ultrastructural mechanisms of transendothelial trans port in blood vessels. Eksp Med Morfol 1980; 19: 189-196.

61. Wagner HJ, Pilgrim Ch, Brandt J. Penetration and removal of horseradish peroxidase injected into the cerebrospinal fluid: Role of cerebral perivascular spaces, endothelium and microglia. Acta Neuropathol 1974; 27: 299-315.

62. Westergaard E. The blood-brain barrier to horseradish peroxidase under normal and experimental conditions. Acta Neuropathol 1977; 39: 181-187.

63. Westergaard E, Brightman MW. Transport of protein across normal cerebral arterioles. J Comp Neurol 1973; 152: 17-44.

64. Westergaard $\mathrm{E}$. The effect of serotonin, norepinephrine, and cyclic AMP on the blood-brain barrier. J Ultrastruct Res 1975; 50 383-384.

65. Zumkeller M, Dietz H. Ultrastructural changes in the blood-brain barrier in rats after treatment with nimodipine and flunarizine. A comparison. Neurosurg Rev 1996; 19: 253-260. 